\title{
RORSCHACH RESPONSES OF INDIVIDUALS SENSITIVE TO STRESS INDUCED BY EXTREME PERCEPTUAL-MOTOR CONFLICT*
}

\author{
CAROL H. AMMONS \& R. B. AMMONS \\ Psychological Reports \& Montana State University
}

Investigators interested in the problem of validaing Rorschach's test have attempted to develop independent measures of emotional stability. Among others, a number of mirror drawing indices have been proposed. Snoddy (11) utilized a mirror-drawing score, called "the basic endurance capacity score" $(11$, p. 25$)$, with which he found it possible to differentiate the degree of endurance under stress of various "psychopathic states." Since that time Snoddy's general finding has been verified by others. Wechsler and Hartogs (12) reported lowered mirror-drawing performance (higher time and error scores) by persons with greater anxiety. It was possible to discern several distinctive characteristics of the performance of $300 \mathrm{Ss}$, "ranging from mild personality disturbances to severe neurosis and forthright psychosis" (12, p. 622). Significant differences between the mirror-drawing performance of normals and that of psychiatric patients led Louttit to observe that "certain persons are calm and straight-forward in their attack. Others are evidently emotionally disturbed, they are tense, make excessive movements, often concentrate at one point, may verbalize, and may even refuse to continue" (10, p. 284). Although it was not possible to study the personality traits of the individuals involved, Louttit accepted his data as evidence that "subjects who show disturbance on the simple task presented by mirror-tracing will probably show disturbance when required to make other unfamiliar adjustments under pressure in non-laboratory situations" (10, p. 284).

Consistent reports of this nature have led to the conclusion that the "most essential factors" tapped in mirror-drawing performance are "(a) relative emphasis on speed and accuracy of performance, (b) marked agitation and disorientation, (c) degree of endurance under stress, and (d) adaptability to novel situations" (1, p. $454 \mathrm{ff}$.).

Since the degree of sensitivity to stress of established clinical groups is reflected in mirrordrawing performance, it should be possible at least roughly, to identify Rorschach factors which are related to this sensitivity. Brower $(8)$ has investigated the relationship of personality characteristics as measured by the Rorschach to disorientation "under experimentally induced visuo-motor conflict." He found that poor mirror-drawing scores were associated with eccentricity (Dd and S \%), lowered intellectual level (W:M), and neuroticism (FK+ $\mathrm{K}+\mathrm{k}$ ). Stronger "introversial directional trends" in the Rorschach protocols were associated with greater stress scores (lowered performance). However, of the large number of correlations between Rorschach categories and stress scores reported, the majority were quite low and only three of twelve were statistically significant. Important relationships may have been obscured by low scoring reliability as well as by design weaknesses. The study is, nevertheless, interesting and provocative.

* For critical reading of the manuscript, the authors are indebted to Drs. Ernie Meyers and Harold Webster. 
Besides studying differential stress sensitivity in persons already known to be diagnostically different from normal persons, one can also induce stress in "normal" subjects. The observed changes in their behavior may then be related to factors in their Rorschach findings. Two studies have used this approach. Williams (14) had Ss do the Digit Symbol Test before and after being observed by psychologists and shocked electrically at intervals (when performance was supposedly poor). Average decrement in Digit Symbol Test scores under stress and $\mathrm{F}^{+} \%$ on all 10 cards correlated -.60 , which was significant at beyond the one per cent level. Average decrement correlated with $\mathrm{F}^{+} \%$ on color cards -.72 ; Form and Color indices correlated +.35 . Williams concludes that "poor integration of form and color in the Rorschach [are associated with] poor control and non-efficient mental activity under emotional situations" (14, p. 28). F+\% was found by Baker and Harris (4) to correlate +.45 with speech intensity under stress. FC integration, an index of emotional stability, correlated +.42 ("just under significance"). They concluded that lack of adult control of emotions (as indicated in the Rorschach by poor use of color and lack of attentive perceptual discrimination) is associated with less stable behavior under stress.

In the studies in which the Rorschach was actually employed, there appears to have been no attempt to evaluate or report all portions of the protocol, or describe what factors are common to the persons under stress. Since it would be clinically valuable to know what Rorschach signs are characteristic of otherwise "normal" but stress-sensitive persons, it is the purpose of the present paper to attempt such a description.

\section{Procedure}

Subjects. Eight undergraduates and one graduate student served as Ss. Two were female; seven male. Their mean age was 24.6 years, with a range of 21 to 30 years. All had been identified in a previous study (3) of 129 college students as sensitive to stress, this being defined by their inability to follow instructions for mirror-drawing, evidenced by extreme motor disturbance. Ss had been instructed to move the full length of one limb of a six-pointed star pattern either in time with a metronome or at their own rate ${ }^{\star}$ No more than 12 moves for each pattern were permitted. Each $S$ was allowed five attempts separated by much encouragement and correction of errors before he was judged unable to perform the task. A more detailed description of this procedure may be found elsewhere $(2,3)$.

Testing. Rorschach's Ink Blot Test was administered following Beck's procedure (5). The only known deviation from these conditions occurred with subject $M a$ with whom it was necessary to stop after the presentation of Card I for a rather lengthy period of reassurance before the testing could proceed. The Rorschachs were administered several months after the experience with the mirror-drawing task so that the Ss would not connect the two experiences. No Rorschach protocol could be obtained from a tenth $S$, therefore, he was not included in the present analyses.

Scoring. Two persons ${ }^{\star}$ scored all of the protocols independently. The only information provided the scorers was the age and sex of each of the $S$ s. They were asked to score each record according to Beck's system $(5,6)$. The purpose of the study, as far as the scorers were concerned, was to estimate reliability of the various scoring categories. The actual scoring on which the results of the study are based, is a reconciliation by the authors of the two independent scorings.

* Two of the $9 S$ s had been instructed to work at their own rate, 4 Ss at the rate of one move every two seconds, 3 Ss at the rate of one move every second.

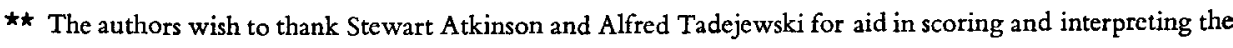
protocols. 


\section{RESULTS}

Number of Responses and their Location. From Table I it can be seen that the number of

Table I. Location Categories, Approach, Sequence, and Time.

\begin{tabular}{|c|c|c|c|c|c|c|c|c|c|c|c|c|c|c|c|c|}
\hline Subject & Sex & $\begin{array}{l}\text { Age } \\
\text { (yr.) }\end{array}$ & $\begin{array}{l}\text { Number } \\
\text { Responses }\end{array}$ & W & DW & $\mathrm{D}$ & $\mathrm{Dd}$ & $\% \mathrm{~W}$ & $\% \mathrm{DW}$ & $\% \mathrm{D}$ & $\% \mathrm{Dd}$ & $\mathrm{d} S$ & Approach & Sequence & $\frac{\text { Time }}{\text { Resp. }}$ & $\frac{\bar{T}}{1 R}$ \\
\hline $\mathrm{Ma}$ & $F$ & 21 & 13 & 4 & - & 9 & - & 31 & - & 69 & - & 0 & WD & - & $57.5^{\star}$ & $66.7^{\star}$ \\
\hline W & $\mathrm{F}$ & 30 & 25 & 7 & 1 & 17 & - & 28 & 04 & 64 & - & 2 & WD & Irregular & 36.8 & 13.2 \\
\hline $\mathrm{C}$ & $\mathbf{M}$ & 21 & 81 & 6 & - & 55 & 2 & 07 & - & 68 & 25 & 90 & $(\mathrm{~W}) \mathrm{D} \mathrm{Dd}$ ! & Confused & 19.8 & 10.9 \\
\hline $\mathrm{Hi}$ & $\mathrm{M}$ & 24 & 19 & 6 & 1 & 12 & - & 32 & 05 & 61 & - & 1 & WD & - & 65.6 & 33.7 \\
\hline $\mathbf{N}$ & $\mathbf{M}$ & 26 & 66 & 6 & - & 52 & 8 & 10 & - & 78 & 121 & 11( & (W)D Dd & Confused & 20.2 & 7.7 \\
\hline G & $\mathrm{M}$ & 29 & 23 & 2 & - & 19 & 2 & 09 & - & 82 & 09 & 20 & (W)D Dd & - & 45.6 & 15.3 \\
\hline $\mathrm{Ha}$ & $\mathbf{M}$ & 23 & 35 & 1 & 2 & 29 & 3 & 02 & 06 & 83 & 09 & 5 & $\mathrm{D} \mathrm{Dd}$ & - & 22.0 & 11.9 \\
\hline$M$ & $\mathbf{M}$ & 25 & 33 & 15 & 2 & 14 & 2 & 5 & 06 & 42 & 06 & 4 V & $\begin{array}{l}\text { W!DW } \\
\text { (D)Dd }\end{array}$ & Confused & 42.2 & 14.0 \\
\hline K & $\mathbf{M}$ & 25 & 19 & 5 & - & 14 & - & 26 & 一 & 72 & - & 3 & WD & 一 & 51.4 & 25.5 \\
\hline
\end{tabular}

* Unit is seconds.

responses ranged from 13 to 81 , with a mean of 34.7. All $S$ s gave at least one whole response, as can be seen from the W column; but the percentages varied from 2 to $45 \%$, with an average of $20 \%$. Four of the $9 S$ s gave DW responses; 2 gave one and 2 gave two. All $S$ s gave $\mathrm{D}$ responses; the range of $\mathrm{D} \%$ was 42 to 83 , with a mean of 68 . The actual frequencies were 9 to 55 . Five of the $9 \mathrm{Ss}$ responded to Dd portions of the blots. The Dd\% of these 5 $S$ s ranged from 6 to 25 , with an average of 12 .

Approach and Sequence. Four of the $S$ s, as shown in Table I, had a WD approach, and four others (C, N, G, M) showed a (W)D Dd approach. The remaining $S$ showed a D Dd approach. In the case of five $S$ s, no sequence could be determined either because there was extensive perseveration or because too few responses were given. Three of the other $S$ s gave a confused sequence and one, an irregular sequence.

Time. The time per response for each $S$ is given in Table I. The times ranged from 19.8 seconds to 57.5 seconds, with a mean of 40.1 seconds. The time for the initial response ranged from 7.7 seconds to 66.7 seconds, with an average of 22.1 seconds.

$\mathrm{F}^{+} \%$. In Tables II and III are given the actual frequencies of $\mathrm{F}^{+}, \mathrm{F}$, and $\mathrm{F}^{-}$, and the $\mathrm{F}^{+} \%$, respectively, for each $S$. The range of $\mathrm{F}^{+} \%$ is from 64 to 100 , with a mean of 76

Table II. Frequencies for Beck's Scoring Categories for Determinants Other than Location by Individual $S$.

\begin{tabular}{|c|c|c|c|c|c|c|c|c|c|c|c|c|c|c|c|c|}
\hline \multirow{2}{*}{ Subject } & \multicolumn{15}{|c|}{ Scoring Categories } & \multirow[b]{2}{*}{ Blends } \\
\hline & $\mathrm{F}^{+}$ & $\mathrm{F}^{-}$ & $F$ & $\mathbf{M}$ & C & $\mathrm{CF}$ & FC & YF & FY & VF & FV & $T$ & $\mathrm{TF}$ & FT & Po & \\
\hline $\mathrm{Ma}$ & 6 & 1 & & 2 & 2 & 1 & & 1 & & & & & & & & \\
\hline W & 9 & 3 & & 3 & 2 & & & & 4 & & & & & 1 & & 3 \\
\hline C & 28 & 14 & 1 & 11 & 1 & 2 & 6 & 1 & 5 & & 6 & & & & 1 & 5 \\
\hline $\mathrm{Hi}$ & 7 & 2 & & 2 & & & 1 & 1 & 4 & & & & & 1 & 1 & 1 \\
\hline $\mathbf{N}$ & 32 & 11 & & 1 & & 2 & 5 & 3 & 4 & 1 & 4 & & & 1 & & 2 \\
\hline$G$ & 13 & 5 & 2 & 1 & & 1 & 1 & 3 & 4 & 1 & 1 & & & & 1 & 3 \\
\hline $\mathrm{H}_{\mathbf{a}}$ & 12 & 4 & 1 & 1 & & & 5 & & 7 & & 3 & & & 1 & & 1 \\
\hline $\mathbf{M}$ & 11 & 4 & 2 & 2 & 2 & 2 & 4 & & & & 1 & 1 & 1 & 1 & & 2 \\
\hline $\mathbf{K}$ & 5 & & & 2 & 1 & 1 & 1 & 1 & 6 & & & & & & & 2 \\
\hline
\end{tabular}


Table III. Factors Indicating Control of Emotional Resources.

\begin{tabular}{cccccc}
\hline Subject & $\mathrm{F}^{+} \%$ & $\mathrm{P}^{\star}$ & $\mathrm{M} / \mathrm{C}$ & Affective Ratio & Lambda Index \\
\hline $\mathrm{Ma}$ & 85 & 4 & $2: 4$ & 116 & 85 \\
$\mathrm{~W}$ & 75 & 10 & $5: 4.5$ & 128 & 108 \\
$\mathrm{C}$ & 64 & 8 & $13: 10$ & 161 & 90 \\
$\mathrm{Hi}$ & 78 & 0 & $2: 1$ & 137 & 122 \\
$\mathrm{~N}$ & 74 & 8 & $1: 7$ & 84 & 53 \\
$\mathrm{G}$ & 72 & 4 & $2: 3$ & 130 & 80 \\
$\mathrm{Ha}$ & 75 & 5 & $1: 3$ & 191 & 105 \\
$\mathrm{M}$ & 73 & 5 & $2: 85$ & 230 & 94 \\
$\mathrm{~K}$ & 100 & 6 & $3: 3$ & 171 & 280 \\
\hline
\end{tabular}

* P stands for Popular Response. Each number is a frequency.

percent. Since the normal range according to Beck (5) is 60 to 90 , with a mean of 83 percent, seven of these $S$ s are within the normal range, one $(K)$ is too high, and one $(C)$ is rather close to the lower limit.

Popular Responses. As shown in Table III, two of the present group (C, N) fall within the normal range of 7 to 9 such responses, one (W) is above and six are well below. Hi gave no popular responses.

Movement Response. Table II shows that all $S$ s gave at least one movement response, one $S(\mathrm{C})$, giving eleven.

Color Responses. All Ss (except Hi) gave two or more responses in which color was an important determinant. Six $S$ s gave responses in which color was more important as a determinant than form; five of these gave several pure color responses. But most color responses were controlled by form for three $S$ s ( $\mathrm{C}, \mathrm{N}$, and $\mathrm{Ha}$ ). When $\mathrm{C}+\mathrm{CF}$ scores were compared with the number of FC for each $S$, three $S$ s $(\mathrm{Ma}, \mathrm{W}, \mathrm{K}$ ) showed fewer FC than $C+C F$ responses; two $S s(G, M)$ had equal frequencies; and five $S$ s had a greater number of FC responses.

Shading. All but one of the Ss (M) responded to the shading, most of them rather often, at least more often than to color $(5 \mathrm{Ss})$. The modal number of responses to shading was seven. Five $\mathrm{Ss}\left(\mathrm{W}, \mathrm{C}, \mathrm{Hi}, \mathrm{Ha}, \mathrm{K}\right.$ ) gave more $\mathrm{FY}$ responses than $\mathrm{Y}+\mathrm{YF}^{+}$blend responses. $\mathrm{N}$ was the only $S$ who had a greater frequency of the less well controlled responses. (See Table II.)

Vista and Texture Responses. Seven $S$ s gave vista responses; one $S(\mathrm{C})$ gave eight; and two gave one relatively less-controlled vista response. One or two of these responses for each $S$ was scored in a blend. Eight $S$ s gave blend responses. There was only one $S(M)$ with a pure texture response, but five $S$ s altogether gave one or more texture-determined responses. (See Table II.)

Position. Subjects $\mathrm{C}, \mathrm{Hi}$, and $\mathrm{G}$ each gave one response which was primarily determined by the position of the blot area (See Table II.)

Experience Balance, $M / C ;$ Affective Ratio; and Lambda Index. In Table III are given three indices of emotional balance. $\mathrm{M} / \mathrm{C}$ ratios are weighted toward color for five $S \mathrm{~s}$; toward movement experience for three; and toward neither for one. The Affective ratios range from 84 percent to 230 percent, with a mean of 149 percent. Since Beck (6) reports the average normal ratio to be $62.45 \pm 25.6$, these ratios are well above the normal range with the 
exception of $\mathrm{N}$ whose ratio falls at the top limit of the normal range. The Lambda Index ranges from 53 to 280 percent, with a median of 94 percent. These ratios appear to be more spread out than those for the Affective Ratio, indicating varying degrees of "adhesion to conscious control" $(5, \mathrm{p} .154)$. It was noted that the sum of all non-F scored responses ranged from $35(\mathrm{~N})$ to $73(\mathrm{~K})$ percent, with a mean score of 52 percent. $K$ and $G$ gave scores of 73 and 69 percent, respectively; the remaining six $S$ s scored between 40 and 57 percent.

White Space Responses. These ranged in number from zero to eleven, with a mean frequency of four. (See Table I.)

Change of Tempo. Each protocol was examined for changes in number of responses per card, time for initial response, and frequency of $\mathrm{F}^{+}$responses per card. All records showed irregular times, six also showed irregularities in frequency of response to cards, and three $S$ s gave irregular numbers of $\mathrm{F}^{+}$responses. Perseveration of a particular kind of irregular kind of irregularity (usually time for first response) over two (and sometimes four) cards appeared in all nine protocols.

Content. Table IV shows the frequencies for each of the content categories described by

Table IV. Frequency of Content Categories for Each of Nine Ss.

\begin{tabular}{|c|c|c|c|c|c|c|c|c|c|c|c|c|}
\hline \multirow{2}{*}{ Subject } & \multicolumn{12}{|c|}{ Content Category } \\
\hline & $\mathrm{H}$ & $\mathrm{Hd}$ & A & Ad & $\overline{A n}$ & $\mathrm{~A}$ & A & Art & $\mathrm{Hh}$ & $\mathrm{Bt}$ & $\mathrm{BI}$ & $\mathrm{Cg}$ \\
\hline $\mathrm{Ma}$ & 1 & 1 & 5 & 1 & & \multirow{2}{*}{\multicolumn{2}{|c|}{1}} & 3 & & & & 1 \\
\hline W & 7 & 7 & 3 & & & & & 1 & & \multicolumn{2}{|l|}{1} & \\
\hline C & 20 & 21 & 17 & 8 & & & & 2 & 1 & & & \\
\hline $\mathrm{Hi}$ & & 3 & 12 & 2 & 1 & & & & & & & \\
\hline $\mathrm{N}$ & 2 & 3 & 22 & 8 & 4 & & & 2 & & \multirow[t]{2}{*}{2} & \multirow[t]{2}{*}{1} & 1 \\
\hline $\mathrm{G}$ & 2 & 4 & 7 & 2 & 1 & & & 1 & & & & \\
\hline $\mathrm{Ha}$ & & 8 & 7 & 6 & 8 & & & & & 3 & & \\
\hline $\mathbf{M}$ & 3 & & 7 & 4 & 1 & & 1 & 3 & 1 & 1 & 1 & 2 \\
\hline $\mathrm{K}$ & 2 & 1 & 6 & 2 & 1 & & & & & & 1 & 1 \\
\hline \multirow{2}{*}{ Subject } & \multicolumn{12}{|c|}{ Content Category } \\
\hline & $\mathrm{Cl}$ & $\mathrm{Ge}$ & $\operatorname{Imp}$ & & $s$ & $\mathrm{Mu}$ & $\mathrm{Na}$ & $\mathrm{Pr}$ & $\mathrm{Rc}$ & $\mathrm{Rl}$ & $\mathrm{Sc}$ & $\mathrm{Tr}$ \\
\hline \multicolumn{13}{|l|}{$\mathrm{Ma}_{2}$} \\
\hline W & & & & & 4 & & 2 & 1 & & & & \\
\hline C & & & 1 & 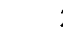 & 2 & & & & & 2 & 1 & 3 \\
\hline $\mathrm{Hi}$ & & & & & & & & & & & & \\
\hline $\mathrm{N}$ & 5 & 3 & & 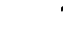 & 7 & 2 & 2 & & 2 & & & \\
\hline G & & 1 & & 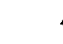 & 7 & & 1 & & & & & \\
\hline $\mathrm{Ha}$ & & & & 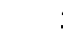 & 3 & & & & & & & \\
\hline $\mathrm{M}$ & & 3 & 1 & 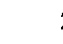 & 2 & & 1 & 1 & & & & 1 \\
\hline $\mathrm{K}$ & 2 & 1 & 1 & 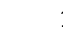 & & & & & & & & \\
\hline
\end{tabular}

* Abbreviations are the same as those used by Beck $(5,6)$.

Beck (5). Of the nine $S$ s, seven (all but $\mathrm{Hi}$ and $\mathrm{Ha}$ ) gave human $(\mathrm{H})$ responses, and seven (excepting W and $\mathrm{M}$ ) gave human detail responses (Hd). Nine $S$ s also gave both animal (A) and animal detail (Ad) responses. Six Ss used anatomy (An) descriptions. Landscapes (Ls) were described by all but one $S(\mathrm{Ma})$. Use of other caregories varied from $S$ to $S$. The average number of categories for all $S$ s was 10 , but this varied from 5 or $6(\mathrm{Hi}, \mathrm{Ha})$ to as many 
as $15(\mathrm{~N}, \mathrm{M})$. There were some responses which had secondary content. $\mathrm{C}$ gave four fire responses and $\mathrm{K}$ gave two responses which were scored both smoke and ink.

Two additional indices for each $S$ were obtained. The first was $\mathrm{H} / \mathrm{Hd}$ ratio which indicates degree of "intrapsychic constriction." One $S(\mathrm{Ma})$ showed equal numbers of $\mathrm{H}$ and $\mathrm{Hd}$ responses; three (W, M, K) gave a greater frequency of $\mathrm{H}$ responses; and five $S$ s had more $\mathrm{Hd}$ than $\mathrm{H}$ responses. According to Beck (6), normal persons have a clear excess of $\mathrm{H}$ over $\mathrm{Hd}$ responses. It will be noticed in table IV that two $S$ s gave no human responses, and two gave no human detail responses. The second index calculated was percent animal responses $(A \%)$. These percentages ranged from 31 to 74 , with a mean of 46 percent. Subjects $C$ and $M$ had low scores; Hi and $K$ had high ones.

Attitude Toward Popular Responses and Sex During Inquiry. Seven Ss rejected popular responses suggested by the examiner during testing of the limits in the inquiry, but three $S$ s (W, C, K) were able to accept one or more. Only $\mathrm{G}$ was able to accept both female and male sexual symbols, but three of the male $S_{s},(\mathrm{Hi}, \mathrm{K}, \mathrm{N})$ were able to point out possible female symbols.

The card most frequently disliked was V (by six $S_{\mathrm{s}}$ ), although I, VI, and VII were not liked by one $S$ apiece. The cards most frequently liked were III (W, C, M), VII (C, Hi, Ha), and X (Ma, N). Ma rejected Card VI; Hi rejected Card II; and G rejected Card III.

All $S$ s but $\mathrm{K}$ described colors on one or more of the cards, $W$ six times and Hi eight times.

Shock. Using Beck's criteria for the several kinds of shock $(5,6)$ there was evidence of color shock in the protocols of $\mathrm{Ma}, \mathrm{W}, \mathrm{C}, \mathrm{Hi}, \mathrm{N}$, and $\mathrm{K}$. Clear indication of shading shock appeared in the records of $\mathrm{W}$ and $\mathrm{N}$; and texture shock in the records of $\mathrm{K}$ and Ma. Shock, probably sexual in nature, appeared in six records ( $\mathrm{Ma}, \mathrm{W}, \mathrm{Hi}, \mathrm{Ha}, \mathrm{M}$, and $\mathrm{K}$ ).

\section{Discussion}

The findings reported in the above section indicate that these $S$ s, like those studied by previous investigators of stress $(4,8,10,12,14)$, show characteristic personality difficulties. Several outstanding neurotic characteristics of the protocols can be identified. First, the functional level of intellectual performance is lowered, impaired by the presence of affect which is difficult to control. Emotionality, released by color, reduces the ability to perform in the stress situation and in the testing conditions. In the latter situation, tempo is upset; sequence is confused or perseveration so marked that no sequence can be described; and the characteristic approach involves orientation toward the obvious, with too much or too little attention to detail or over-all organization.

Secondly, this group shows great sensitivity to excitement. Their own personal needs are so strong that they are easily elicited by the testing materials. Sometimes, however, these $S$ s are likely to be overwhelmed by the intensity of their experiences, and even become relatively insensitive to potential dangers, as a result. The sensitivity is indicated by a number of Rorschach factors (marked irregularities in sequence, changes of tempo, high A\% and Affective ratios), and openly expressed as pleasure in or identification of the colors of the cards. Beck (6) also suggests that high sums of responses which do not have form as a determinant (non-F responses) reflect the enormity of emotional experience characteristic of 
neurotics. There are relatively high frequencies of responses whose content is scored anatomy and blood. Marked evidence for neurotic shock according to Beck's criteria $(5,6)$, related to both color and shading, is found in every record.

The intensity of their emotional experiences probably has led these $S$ s to make efforts at control shown in the records. These college students have sought in one of two general ways to handle the anxiety arising from their sensitivity to stimulation. Some are hyperactive, appearing rebelliously opposed to their environment, and show evidence of moving away from others. These $S s$ show high "intrapsychic constriction" (H/Hd), low $P$ frequency, high $\mathrm{A} \%$, and a high incidence of $\mathrm{S}$ responses. Most of the $S \mathrm{~s}$, however, appear to be reactively depressed. They tend to have a high incidence of vista responses, increased concern with their own bodies, lowered total number of responses, too low or too high $\mathrm{F}^{+} \%$, and more $\mathrm{M}$ than $\mathrm{C}$. All of these $S \mathrm{~s}$ tend to qualify or deny their responses. There is perseveration from card, and within a series of responses to one card. This apparent inability to attend to new concepts or card areas may be related to the "freczing" behavior observed in the mirror-drawing performance of these $S$ s and other disturbed persons $(10,11,12)$. Freezing appears most frequently at the start of the first move on a new pattern or where a change in direction of movement is required. $S$ is not familiar with the effect of the mirror on apparent direction of movement and seems to be reluctant to try "new" ways of moving, particularjy when he is very likely to make errors (i.e., get off the pattern). In place of making errors, he initiates many small movements but quickly inhibits them, in effect remaining at one point on the pattern. In perseveration during the Rorschach, the $S$ 's repetition of one response or one way of responding may also reflect the inhibition of response tendencies associated with conflict.

There are some trends toward much greater disturbance in several protocols, such as position responses, four or five contaminations, and excessive perseveration. In spite of this, these students were all managing to do their school work satisfactorily, could respond to a good many social situations and for the most part had a fairly wide range of interests. Their overt behavior in the testing situation showed at times rather acute awareness of their own distress.

Although nearly all authors conclude that stress tends to impair performance, particularly that of disturbed persons, it is in many cases not possible to compare the data which they report with those in the present study. Many studies did not use the Rorschach (10, 11) or employed a less widely used scoring method as in the case of Brower's investigation (8). Williams (14) and Baker and Harries (4) simply reported significant positive correlations between decrements in performance under stress and $\mathrm{F}^{+} \%$ and between the total number of responses and performance under stress (14). Both interpret their findings as indicative of lack of or poor emotional control. Westrope (13) also gave only one index (lowered total number of Rorschach responses) and commented that the other indices did not differentiate between her anxious and non-anxious groups under stress conditions.

Rorschach findings are reported in greater detail in a study by Berger (7) of stress experienced at admission to a tuberculosis sanatorium as compared with later retest performance. He used Beck's method of scoring and, although he reported only test-retest difference scores, there is information about several different scoring categories. Comparison of his 
results with those of the present study shows the following areas of agreement: (a) heightened $\mathrm{F}^{+} \% \mathrm{~b}$ under stress; (b) smaller total number of responses; (c) increased reaction time (T/IR); (d) lessened creativity as indicated by $M$; (e) heightened dysphoric and inferiority feelings; $\Sigma \mathrm{Y}$ and $\Sigma \mathrm{V} ;(\mathrm{f})$ increased stereotypy and evasiveness, $\mathrm{A} \%$; (g) lessened ability to think in terms of group standards, $\mathrm{P}$, and finally; (h) a markedly increased number of rejections. Berger's finding of decreased breadth of interest (indicated by content scores) may not be considered as contradicted by the present results, because the educational level and socio-economic background of the $S$ s in the two studies were very different. He also reported that stress increased whole responses, while decreasing obvious large detail (D) responses. This discrepancy is not so easily resolved. In the present study it was found that three of a small group of stress-sensitive persons tended to underemphasize whole responses, whereas one clearly overemphasized them. Five $S$ s tended markedly toward the obvious, i.e., gave a greater proportion of $\mathrm{D}$ responses,

From this discussion it would seem obvious that there is a real need for careful systematic evaluation of the various Rorschach categories, using larger groups and comparing performance with induced stress with that of clinically sensitive persons.

\section{SUMMARY}

Nine Ss, who had shown themselves to be overly sensitive to stress in a mirror-drawing situation, werc given Rorschach's Ink Blot Test. Analysis of the results, according to Beck's system, indicated that these stress-sensitive individuals were extremely neurotic. Poorly controlled emotionality was high and tended to reduce their productivity. Many $S$ s appeared to feel overwhelmed by the intensity of their experiences, attempting to handle their anxiety by opposing the environment actively or by becoming reactively depressed.

\section{REFERENCES}

1. Abt, L. E., \& Bellak, L. Projective psychology: clinical approaches to the total personality. New York: Knopf, 1950.

2. Ammons, C. H. A method for pacing movements in mirror drawing. Mot. Skills Res. Exch., 1950, 2, 59-62.

3. Ammons, C.H. The effects of pacing and duration of interpolated rest on mirror drawing performance. J.gen. Psychol., in press.

4. Baker, L.M., \& Harris, J. S. The varidation of Rorschach test results against laboratory behavior. J. clin. Psychol., 1949, 5, 161-164.

5. Beck, S.J. Rorschach's test. I. Basic processes. New York: Grune \& Stratton, 1949.

6. Beck, S.J. Rorschach's test. II. A variety of personality pictures. New York: Grune \& Stratton, 1946.

7. Berger, D. The Rorschach as a measure of real-life stress. J. consult. Psychol., 1953, 17, 355358.

8. Brower, D. The relations of visuo-motor conflict to personality traits and cardiovascular activity. J.gen. Psychol., 1948, 38, 69-99.
9. Hartogs, R. A contribution to the analysis and and measurement of anxiety. Master's Thesis New York University, 1945.

10. Louttit, C.M. The mirror tracing test as a diagnostic aid for emotional instability. Psychol. Rec., 1943, 5, 279-286.

11. Snoddy, G.S. Learning and stability. A psychophysiological analysis of a case of motor learning with clinical applications. Quantitative and clinical aspects. J. appl. Psychol., 1926, 10, 1-36.

12. Wechslet, D., \& Hartogs, R. The clinical measurement of anxiety. Psychiat. Quart., 1945, 19, 618-635.

13. Westrope, M.R. Relations among Rorschach indices, manifest anxiety and performance under stress. $J$. abnorm. soc. Psychol., 1953, 43, 515-524.

14. Williams, M. An experimental study of intellectual control under stress and associated with Rorschach factors. J. consult. Psychol., 1947,11, 21-29.

MS. reccived X 10,60 . 